$\mathrm{E}$

EVALUAR
2017, Vol. 17, No 2 .

ISSN 1667-4545

Recuperado de https://revistas.unc.edu.ar/index.php/revaluar

Laboratorio de Evaluación Psicológica y Educativa

Facultad de Psicología - Universidad Nacional de Córdoba

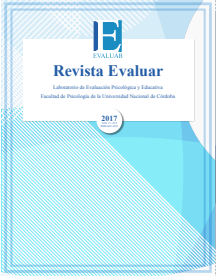

\title{
Matrices Progresivas de Raven: Percentiles para Preescolares Cubanos de entre 4 y 6 años
}

\author{
Yaser Ramírez-Benítez * 1, Bárbara Bermúdez-Monteagudo ${ }^{2}$, Shuyeng Acea-Vanega ${ }^{3} \mid$ Introducción \\ Método \\ 1 - Universidad de Cienfuegos, Cuba. \\ 2 - Universidad de Cienfuegos, Cuba. \\ 3 - Sala de Rehabilitación del Policlínico Ramón Claudio Delgado Amistoy, Cienfuegos, Cuba. \\ Resultados \\ Discusión \\ Referencias
}

Recibido: 07/06/2017 Revisado: 26/06/2017 Aceptado: 14/07/2017

\section{Resumen}

La prueba Matrices de Raven es una alternativa para evaluar la inteligencia en niños en edad preescolar. La investigación se propuso determinar percentiles en la prueba Matrices de Raven para niños cubanos de 4 a 6 años, validar percentiles con respecto a un punto de corte cubano (Ramírez-Benítez et al., 2015) y determinar las propiedades psicométricas de la prueba desde un análisis de ítems. Participaron 803 niños (380 varones) seleccionados en 15 instituciones educativas de enseñanza regular en las ciudades de Cienfuegos y Matanzas, Cuba. El análisis de validez entre el punto de corte cubano y los percentiles obtenidos indicó que los percentiles deben utilizarse en la práctica con precaución. No obstante, los percentiles clasifican con mayor flexibilidad al niño con alteraciones en el neurodesarrollo con respecto al punto de corte cubano de Ramírez-Benítez et al. (2015). En resumen, los percentiles son adecuados para clasificar al niño preescolar cubano, aunque requieren de otros estudios de validez.

Palabras clave: Matrices de Raven, inteligencia fluida, preescolar, neurodesarrollo

\begin{abstract}
The Raven Progressive Matrices Test is an alternative to assess intelligence in preschool children. This study attempted to determine percentiles in the Raven Matrices Test to assess Cuban children between 4 and 6 years old, to validate these percentiles regarding a Cuban cut-off point (Ramírez-Benítez et al., 2015) and to determine the psychometric properties of the test through item analysis. A total of 803 children ( 380 boys) were selected in 15 regular educational institutions in the cities of Cienfuegos and Matanzas, Cuba. The validity analysis between a Cuban cut-off point and the obtained percentiles indicated that these percentiles should be used in practice with caution. However, percentiles allow for a more flexible assessment in children with neurodevelopmental disorder than the Cuban cut-off point from Ramírez-Benítez et al., 2015. Evidence supports adequacy of percentiles to assess Cuban preschool children, although further validity studies are required to support these findings.
\end{abstract}

Keywords: Raven's Matrices Test, fluid intelligence, preschool children, neurodevelopment 


\section{Introducción}

El estudio de la inteligencia es uno de los capítulos más leídos e investigados en la historia de la psicología. El enfoque psicométrico ha sido el modelo teórico que más investigaciones ha generado sobre la inteligencia, y al mismo tiempo ha formulado varias preguntas que aún permanecen $\sin$ respuesta.

En la década de los '90, la Asociación Americana de Psicología publicó un informe oficial sobre los conocimientos y el desconocimiento de la inteligencia humana (Neisser et al., 1996), el cual es de obligada consulta para los profesionales de la conducta. Según el comité redactor de ese informe, presidido por Ulric Neisser, la inteligencia se puede medir, y los tests de inteligencia la miden adecuadamente. Dichos tests constituyen el modo de evaluación de la inteligencia más preciso, confiable y adecuado, y tienen múltiples aplicaciones y utilidades.

En dicho informe se reconoce la importancia de la teoría del factor $g$ de Spearman como la teoría de la inteligencia más conocida de todos los tiempos, pues ha posibilitado el diseño de varias pruebas de inteligencia en diversos contextos culturales. De acuerdo con esta teoría, la inteligencia puede ser explicada en función de un factor de capacidad mental general $g$ y de un gran número de factores específicos $s$ (Spearman, 1904). El factor $g$ es una habilidad fundamental que interviene en todas las operaciones mentales, representa la energía mental y se pone en funcionamiento en toda tarea no automatizada. Es una capacidad de reflexión que permite al sujeto observar lo que ocurre en su interior, concebir las relaciones esenciales existentes entre dos o más ideas (educción de relaciones) y captar las ideas implícitas en una relación (educción de correlatos).

Las tareas cognitivas más cargadas de $g$ son aquellas que exigen razonamiento deductivo o inductivo, visualización espacial, razonamiento cuantitativo, y conocimiento y razonamiento verbal (significado de las palabras, distinciones entre palabras relacionadas, sinónimos-antónimos, analogías verbales y comprensión lectora; Crone et al., 2009). Entre los principales instrumentos que miden el factor $g$ se encuentran las Matrices Progresivas de Raven (Raven, 2004), el Test del Dominó (Anstey, 1963) y los Tests de Factor $g$ de Cattell (Cattell \& Cattel, 1994).

El Test de Matrices Progresivas Coloreadas de Raven (TMCR) es un instrumento adecuado para medir el factor $g$ en edades infantiles, y presenta particularmente tres grandes atractivos: (1) es una prueba fácil de aplicar y su ejecución requiere de poco tiempo; (2) puede ser aplicado en poblaciones de niños con alteraciones del neurodesarrollo, en particular en niños con afectaciones del habla (sordera, hipoacusia, afasias, autismo), y (3) las puntuaciones tienen una correlación significativa con el aprendizaje, en especial en los primeros años de vida (Ramírez-Benítez, Torres-Diaz, \& Amor-Diaz, 2016).

Desde esta perspectiva, el TMCR tiene una amplia utilidad en contextos clínicos y educativos; sin embargo, desde el año 2000 hasta la fecha, varios grupos de investigación han realizado publicaciones en las que se manifiestan a favor de actualizar las normas de dicha prueba, específicamente para las edades comprendidas entre 6 y 60 años (Bandeira, Boccato-Alves, Giacomel, \& Lorenzato, 2004; Fernández-Liporace, Ongarato, Saavedra, \& Casullo, 2004; Fernández-Nistal \& Mercado-Ibarra, 2014; Ivanovich et al., 2000; Mansilla, Vásquez, \& Estrada, 2012; Rossi-Casé et al., 2014, 2015, 2016). Los investigadores justifican la actualización de la norma por tres razones: (1) la necesidad de realizar interpretaciones diagnósticas adecuadas; (2) la necesidad de realizar revisiones periódicas de las pruebas psicométricas en función de los modelos teóricos ac- 
tuales o cambios culturales en el contexto, y (3) necesidad de revisar las puntuaciones directas de la prueba debido a la inconsistencia en los resultados que podría ocasionar el efecto Flynn.

En relación con el efecto Flynn, este hace alusión al hecho de que la norma utilizada para evaluar a los sujetos en una población dada se hace obsoleta conforme pasan los años, pues se espera una puntuación mayor a medida que pasa el tiempo. De esta forma se hace necesario realizar investigaciones que permitan modificar las normas y clasificar de manera adecuada la inteligencia.

El aumento de las puntuaciones es mayor en aquellos tests que miden la inteligencia fluida (pruebas de inteligencia libres de influencia educativa) que en las que miden la inteligencia cristalizada (Sundet, Barlaug, \& Torjussen, 2004). Esto llevó a pensar que el aumento estaba relacionado con el factor $g$ de la inteligencia, ya que las pruebas del factor $g$ tienen una mayor correlación con las tareas de inteligencia fluida comparadas con factores específicos como las pruebas de vocabulario, cálculo y lectura.

La causa de este fenómeno se desconoce hasta el momento, pues el aumento de los puntajes directos no es sinónimo de aumento de la inteligencia en la población; además, las evidencias presentadas hasta el momento no son suficientes para afirmar que la inteligencia de la población esté aumentando. Una explicación posible de este fenómeno está relacionada con el entrenamiento vinculado a diversos factores culturales y educativos tales como el incremento de la educación desde edades tempranas y el acceso directo a diversas tecnologías digitales desde edades tempranas (Rossi-Casé et al., 2014).

Una reciente revisión del tema evidencia que el efecto Flynn ocurre en algunas poblaciones de países desarrollados (Flynn \& Rossi-Casé, 2012; Rossi-Casé et al., 2015) mientras que otros resultados no confirman tal efecto, pues destacan que los valores disminuyen con el transcurso de los años o bien que existe un efecto meseta para las puntuaciones obtenidas (Bandeira, Costa, \& Arteche, 2012; Flynn, 2013; Rossi-Casé et al., 2014). El principal debate se centra en los países desarrollados, donde se espera un efecto Flynn debido a la influencia del nivel económico, el grado educacional y los niveles de vida. Sin embargo los resultados no son consistentes. En efecto, si en un grupo de países desarrollados no ocurre el efecto Flynn, lo cual constituye la minoría de los casos, se espera que en el resto de los países subdesarrollados no ocurra tal efecto (Teasdale \& Owen, 2007; Brouwers, Van de Vijver, \& Van Hemert, 2008).

En Cuba se han realizado pocos estudios para proponer una norma del TMCR (Cairo-Valcárcel, Cairo-Martínez, Bouza, \& Solazabal, 2000; Ramírez-Benítez, Jiménez-Morales, \& Diaz-Bringas, 2015); en su lugar se utilizan normas de la población mexicana (Raven, 2004) por la cercanía cultural a nuestro contexto. En forma similar, no se han realizado estudios sobre el efecto Flynn con muestras cubanas. Por ende, el objetivo principal de este trabajo es obtener percentiles para clasificar la inteligencia de manera adecuada. En este sentido, al considerar la aplicación corta y fácil de la prueba, Cairo-Valcárcel et al. (2000) presentaron una propuesta de percentiles con la intención de valorar la inteligencia en la población cubana, aunque la propuesta se focalizó en jóvenes y adultos. Por otra parte, un intento de establecer un punto de corte para conocer el desarrollo del razonamiento analógico en niños preescolares cubanos fue llevado a cabo por Ramírez-Benítez et al. (2015); sin embargo, el uso de un punto de corte posibilita una clasificación basada en dos opciones: desarrollo típico de la edad versus desarrollo insuficiente para la edad. De esta forma, el profesional está limitado 
a clasificar al niño en dos grupos, lo cual es poco habitual en la práctica profesional cuando se trata de un instrumento de inteligencia.

Otros puntos de interés están relacionados con el uso del TMCR en niños en edad preescolar, la actualización de la norma y el análisis de ítems de la prueba para estas edades. Una revisión en la literatura reveló que la mayoría de las actualizaciones de la norma se proponen para la evaluación en sujetos de entre 6 años y edades adultas (Bandeira et al., 2004; Fernández-Liporace et al., 2004; Fernández-Nistal \& Mercado-Ibarra, 2014; Ivanovich et al., 2000; Mansilla et al., 2012; Rossi-Casé et al., 2014, 2015, 2016), lo cual resulta curioso si se compara con el aumento reciente de investigaciones con niños en edad preescolar (Tenorio et al., 2012; Lázaro, Ostrosky, \& Lozano, 2012; Solovieva, Loredo, Quintanar, \& Lázaro, 2013 ) con el fin de detectar alteraciones del neurodesarrollo, en especial entre los 3 y 6 años de edad. En particular, las investigaciones valoran la inteligencia en preescolares a través de las diferentes variantes de la escala de Wechsler (WPPSI-IV, Wechsler, 2011, y WISC-IV, Wechsler, 2012), pues esta escala aporta mayor información al valorar el desarrollo cognitivo del niño. Sin embargo, una estimación rápida del razonamiento analógico podría ser realizada a través del TMCR en niños en edad preescolar, ya que sus puntuaciones muestran una significativa relación con el desempeño de las habilidades escolares, tanto al inicio como al final de la etapa escolar.

Si bien el TMCR está diseñado para niños entre 5 y 11 años, algunos investigadores han demostrado que un niño de 4 años y medio de edad es capaz de resolver algunos problemas de matrices (Ramírez-Benítez et al., 2015; Raven, 2004). Asimismo, Raven (2004) relacionó el desarrollo de la capacidad intelectual con la edad cronológica, y estableció que desde los 4 años el niño puede resolver algunos problemas, pues es capaz de darse cuenta de que a una figura grande le falta una parte y esa parte puede estar dentro de las opciones de respuesta. Así, el TMCR puede ser aplicado desde los 4 años y puede, en consecuencia, estimular el desarrollo del pensamiento, así como detectar alteraciones a edades tempranas.

En relación con el análisis de ítems en la prueba Raven, se puede observar una tendencia similar a la de los estudios de actualización, pues el investigador que realiza un estudio de normalización utiliza también los datos de la muestra para realizar un análisis de ítems. Desde esta perspectiva, se puede observar una carencia de estudios que valoren el rendimiento de los ítems del TMCR en edades preescolares.

Considerando los puntos señalados, este trabajo propone determinar un conjunto de percentiles para la prueba TMCR a fin de clasificar el razonamiento analógico de niños cubanos en edad preescolar, entre 4 y 6 años, así como determinar la validez de los percentiles propuestos con respecto al punto de corte de Ramírez-Benítez et al. (2015). De esta manera, el especialista puede clasificar al niño dentro de un rango de cinco percentiles: percentil 90: niños con puntuaciones superiores para su edad; percentil 75: niños con puntuaciones altas para su edad; percentil 50: niños con puntuaciones típicas para su edad; percentil 25: niños con puntuaciones bajas para su edad, y percentil 10: niños con puntuaciones muy bajas para su edad. Además, este estudio se propone determinar las propiedades psicométricas del TMCR desde un análisis de ítems a fin de valorar tanto la complejidad del instrumento para niños en edad preescolar como la capacidad para discriminar entre niños con baja y alta habilidad en el razonamiento analógico. 
Método

Participantes

Se realizó un diseño de tipo no experimental transversal correlacional, en el cual se utilizaron dos muestras de normalización: la muestra de Ramírez-Benítez et al. (2015) y una muestra no probabilística intencionada aplicada en 2016 (de enero a mayo).

En total, participaron 803 niños (380 varones y 423 niñas) de entre 4 años y 5 meses y 6 años y 11 meses de edad seleccionados en 15 instituciones educativas de enseñanza regular en las ciudades de Cienfuegos y Matanzas, Cuba (8 círculos infantiles y 7 escuelas regulares).

En 2016, se seleccionaron al azar 4 círculos infantiles y 3 escuelas regulares de la provincia de Cienfuegos y se evaluó a todos los niños de entre 4 y 6 años. En los círculos infantiles, los niños fueron evaluados por año de vida: $4^{\circ}$ año de vida: edades comprendidas entre 4 años y 5 meses y 4 años y 10 meses; $5^{\circ}$ año de vida: edades comprendidas entre 5 años y 2 meses y 5 años y 9 meses; y finalmente, $6^{\circ}$ año de vida: edades comprendidas entre 6 años y 2 meses y 6 años y 11 meses. Una parte de la muestra se seleccionó en los círculos infantiles debido a que estas son instituciones donde la población infantil recibe orientación educativa de manera sistemática y organizada. De esta forma, se podía controlar la asistencia y permanencia de los niños en la investigación.

En relación con las escuelas regulares, se evaluaron todos los grupos de $1^{\circ}$ grado con edades comprendidas entre 6 años y 2 meses y 6 años y 11 meses. Además, en las escuelas regulares la matrícula de niños con 6 años es mayor, lo cual justifica el tamaño mayor de la muestra de niños de 6 años con respecto a las demás edades.

El estudio fue autorizado por el Ministerio de Educación Provincial y se contó con el consentimiento de los padres de aquellos niños evaluados.
Se estima que la muestra utilizada corresponde aproximadamente a un $10 \%$ de la población de niños en edad preescolar en Cienfuegos y Matanzas, de acuerdo con el Anuario Estadístico de Salud en el 2013 (Ministerio de Salud Pública, 2013).

\section{Instrumento}

Test de Inteligencia de Matrices Progresivas Coloreadas de Raven (Raven, 2004). Esta prueba consiste en responder 36 problemas de tipo no verbal que se presentan en un cuaderno de papel. Cada uno de ellos consta de una figura incompleta que el niño debe completar. Se presentan seis alternativas de respuesta posibles, una de ellas es la respuesta correcta y el resto son distractores. La prueba está dividida en tres series de doce problemas cada una: A, Ab y B. La complejidad se incrementa progresivamente a lo largo de la prueba y a lo interno de las series de modo que A1 es el ítem más fácil y B12 el más difícil, pero A12 es más difícil que B1. Las funciones cognitivas que se examinan principalmente son: percepción de tamaños, percepción de orientación en el espacio, en una dirección y en dos direcciones simultáneamente, aprehensión de figuras discretas especialmente relacionadas con un todo (Series A y $\mathrm{Ab}$ ), análisis de un todo en sus componentes, capacidad de concebir figuras correlativas y deducción de relaciones (Serie B). Por cada respuesta correcta se otorga un punto y se recoge una puntuación total en la ejecución del test.

\section{Procedimiento}

La prueba se aplicó a todos los niños de la muestra de manera individual por la mañana. La aplicación se realizó en un local tranquilo e iluminado en presencia de la educadora. 
Análisis de datos

Estadística Descriptiva. Se utilizó el programa SPSS versión 21 (IBM, 2012) para determinar la estadística descriptiva por edades: la media, la desviación estándar, la prueba Kolmogorov-Smirnov de bondad de ajuste a la curva normal y el máximo y el mínimo de las puntuaciones en la prueba.

Propiedades psicométricas. La confiabilidad del instrumento se evaluó a través del análisis de ítems mediante el coeficiente alfa de Cronbach cuando el ítem es eliminado y la correlación ítem-total (índice de homogeneidad). Se utilizó el valor de alfa de Cronbach cuando el ítem es eliminado debido a la importancia que tiene cada ítem de la prueba en niños en edad preescolar, pues se espera que algunos ítems sean más difíciles que otros. Este método analiza el coeficiente alfa de cada ítem, por lo que si alfa aumentara significativamente tras eliminar un ítem específico, esto indicaría que la exclusión de ese ítem aumenta la confiabilidad de la escala. Un ítem con valores de alfa significativamente por encima de los demás podría ser candidato a ser eliminado por ser muy complejo o muy fácil. En igual sentido, se utilizó la correlación ítem-total para conocer cuánto aporta cada ítem al resultado final de la prueba. De esta forma, se obtuvo una medida de confiabilidad de los ítems. La correlación ítem-total se clasificó siguiendo las recomendaciones de Ebel y Frisbie (1986): valores mayores que .39, correlaciones excelentes, el ítem se puede conservar; valores entre $.30-.39$, correlaciones buenas, el ítem tiene posibilidades de mejorar; valores entre .20 - .29, correlaciones regulares, el ítem necesita revisión, y valores menores a .20, correlaciones pobres, el ítem se debe descartar o revisar en profundidad.

En igual sentido se obtuvieron los índices de dificultad y de discriminación de los ítems de la prueba, los cuales fueron clasificados según Crocker y Algina (1986): dificultad baja con valores menores o iguales a .80; dificultad media con valores entre .79 y .50 ; y dificultad alta con valores menores que .50 .

Punto de corte. Se obtuvieron cinco percentiles en cada edad para clasificar al niño con baja y alta habilidad en el razonamiento analógico.

Validez de los percentiles. La validez de los percentiles se evaluó a través de tres procedimientos: (1) la coincidencia (cantidad de verdaderos positivos y negativos) al clasificar el rendimiento intelectual entre el punto de corte de Ramírez-Benítez et al. (2015) y los percentiles propuestos; (2) el cálculo del coeficiente de probabilidad diagnóstica (CPD), y (3) la cantidad de casos clasificados con alteraciones en el rendimiento intelectual por los percentiles propuestos en una muestra de niños con alteraciones en el neurodesarrollo (submuestra de validación, 130 niños, de Ramírez-Benítez et al., 2015). Para aplicar los tres procedimientos fue necesario obtener los indicadores de sensibilidad (capacidad del punto de corte para detectar a los casos positivos - niños que presentan alteraciones intelectuales) y especificidad (capacidad del punto de corte para descartar a los casos negativos - niños que no presentan alteraciones intelectuales).

El coeficiente de probabilidad diagnóstica estima cuánto más probable es el resultado de una prueba o punto de corte en personas enfermas en relación con las personas sanas (Moratalla-Rodríguez, 2015). El cociente de probabilidad positivo $(\mathrm{CPD}+)$, con una significativa relación con los valores de la sensibilidad, estima cuánto más probable es obtener un resultado positivo en sujetos con problemas en el desarrollo que en sujetos sin problemas. Se calcula de la siguiente manera: $(\mathrm{CPD}+)=(\mathrm{Se}) /(1-\mathrm{Es})$ donde $\mathrm{Se}$ representa sensibilidad y Es representa especificidad. El cociente de probabilidad negativo $\left(\mathrm{CP}_{-}\right)$, con una 
significativa relación con los valores de la especificidad, estima cuánto más probable es obtener un resultado negativo en sujetos enfermos que en sujetos sanos. Se calcula de la siguiente manera: $(\mathrm{CPD}-)=(1-\mathrm{Se}) /($ Es $)$.

Según Moratalla-Rodríguez (2015) los valores del CPD se clasifican de la siguiente manera:

- CP > 10: incrementos amplios de la probabilidad diagnóstica.

- CP 5 - 10: incrementos moderados de la probabilidad diagnóstica.

- CP 2 - 5: incrementos pequeños de la probabilidad diagnóstica.

- CP 1 - 2: incrementos insignificantes de la probabilidad diagnóstica.

- CP 1: sin cambios de la probabilidad diagnóstica.

- CP 0.5 - 1: descensos insignificantes de la probabilidad diagnóstica.

- CP 0.2 - 0.5: descensos pequeños de la probabilidad diagnóstica.

- CP 0.1 - 0.2: descensos moderados de la probabilidad diagnóstica.

- $\mathrm{CP}<0.1$ : descensos amplios de la probabilidad diagnóstica.

\section{Resultados \\ Estadística descriptiva}

Los valores de la media indicaron que en la medida que incrementaba la edad, los niños aumentan sus puntuaciones en la prueba Raven, tal y como se indica en la Tabla 1.

Con respecto a la desviación estándar, los valores en los niños de 6 años fueron mayores que los de las demás edades. Esto se debió a que 6 niños obtuvieron puntuaciones máximas. Dichos casos fueron detectados a través de un análisis de frecuencia de las puntuaciones obtenidas por edades. Al eliminarse estos 6 casos del análisis se pu- do observar que el valor de DE disminuyó a 1.85.

La media y la moda en cada edad coinciden como se muestra en la Tabla 1, es por ello que los valores de $Z$ en la prueba de Kolmogorov-Smirnov presentan niveles significativos de normalidad: a los 4 años $(Z=4.29$, Sig. .33), a los 5 años $(Z=1.93$, Sig. .20) y a los 6 años $(Z=2.62$, Sig. $.24)$.

\section{Tabla 1}

Estadística descriptiva por edades. Percentiles por edades. Valores de $\mathrm{Z}$ para las tres muestras independientes (4 años, 5 años y 6 años) en la prueba de normalidad Kolmogorov-Smirnov.

\begin{tabular}{|c|c|c|c|}
\hline \multirow{2}{*}{$\begin{array}{c}\begin{array}{c}\text { Variables } \\
\text { sociodemográficas }\end{array} \\
\text { Características de la muestra }\end{array}$} & \multicolumn{3}{|c|}{ Cantidad de Casos } \\
\hline & 4 años & 5 años & 6 años \\
\hline Niños & 97 & 92 & 191 \\
\hline Niñas & 108 & 113 & 202 \\
\hline Total & 205 & 205 & 393 \\
\hline \multicolumn{4}{|l|}{ Provincia } \\
\hline Cienfuegos & 107 & 102 & 203 \\
\hline Matanzas & 98 & 103 & 190 \\
\hline \multicolumn{4}{|l|}{ Instituciones } \\
\hline Círculos infantiles & 205 & 95 & 112 \\
\hline Enseñanza regular & - & 110 & 281 \\
\hline \multicolumn{4}{|l|}{ Prueba Raven } \\
\hline Media & 14.01 & 15.04 & 16.03 \\
\hline Mediana & 14.00 & 15.00 & 16.00 \\
\hline Desviación típica & 1.44 & 1.82 & 2.74 \\
\hline Mínimo & 8.00 & 9.00 & 6.00 \\
\hline Máximo & 17.00 & 23.00 & 30.00 \\
\hline \multicolumn{4}{|l|}{ Percentiles } \\
\hline 10 & 10.00 & 11.00 & 11.00 \\
\hline 25 & 11.00 & 12.00 & 13.00 \\
\hline 50 & 14.00 & 15.00 & 16.00 \\
\hline 75 & 15.00 & 17.00 & 18.00 \\
\hline 90 & 16.00 & 18.00 & 20.00 \\
\hline Z de K-Smirnov & 4.29 & 1.93 & 2.62 \\
\hline Sig. & .33 & .20 & .24 \\
\hline
\end{tabular}


Tabla 2

Índices de dificultad y discriminación de los 36 ítems del TMCR.

\begin{tabular}{lccc}
\hline & \multicolumn{3}{c}{ Índice de Discriminación } \\
\cline { 2 - 4 } & $\begin{array}{c}\text { Buena } \\
\text { (mayor o igual a .20) }\end{array}$ & $\begin{array}{c}\text { Aceptable } \\
\text { (entre .10 y .19) }\end{array}$ & $\begin{array}{c}\text { Mala } \\
\text { (menor o igual a .10) }\end{array}$ \\
\hline $\begin{array}{l}\text { Índice de Dificultad } \\
\text { Baja } \\
\text { (mayor o igual a .80) }\end{array}$ & $8,9,10,13,18,24,25,34$ & $1,2,28,29,30$ \\
$\begin{array}{l}\text { Media } \\
\text { (entre .51 y .79) }\end{array}$ & $4,5,6,7,19,20,21,22,23$, & $3,14,15,16,17$ \\
$\begin{array}{l}\text { Alta } \\
\text { (menor o igual a .50) }\end{array}$ & $11,12,26,33,35$ & \\
\hline
\end{tabular}

Propiedades psicométricas: Análisis de items

Confiabilidad. El coeficiente alfa de Cronbach cuando el ítem es eliminado indicó valores adecuados para la muestra total (entre .84 y .89). El análisis indicó que si se eliminan los ítems $1,4, \mathrm{y}$ 32 alfa aumenta a .89 .

Los valores de alfa también resultaron adecuados por edad. A los 4 años se obtuvieron valores de alfa de entre .82 y .86, con posibilidades de aumentar hasta .86 si se eliminan los ítems $1,9 \mathrm{y}$ 33. A los 5 años los valores de alfa fueron de entre .85 y .88 y no se encontraron ítems con valores destacados; por ende, no se recomienda eliminar ningún ítem. A los 6 años se obtuvieron valores de entre .84 y .90 con posibilidades de aumentar alfa a .90 si se eliminan los ítems 1, 2, 3, 4 y 32 .

En relación con la correlación ítem-total o índice de homogeneidad, las correlaciones oscilaron entre excelentes y buenas con valores de entre .33 y .56 : El $30 \%$ de los ítems obtuvo excelentes correlaciones (ítems 6, 7, 8, 11, 17, 18, 19, 23, $24,28,30)$ y el resto obtuvo buenas correlaciones (ítems 1, 2, 3, 4, 5, 9, 10, 12, 13, 14, 15, 16, 20, $21,22,25,26,27,29,31,32,33,34,35,36$ ).

Índices de dificultad y discriminación. En la Tabla 2 se muestran los índices de dificultad y de discriminación de los ítems de la prueba. En rela- ción con el índice de discriminación, el 72.2\% (26 ítems de 36) de los ítems son buenos para discriminar entre niños con alta y baja habilidad (ítems $4,5,6,7,8,9,10,11,12,13,18,19,20,21,22$, $23,24,25,26,27,31,32,33,34,35$ y 36$)$ y un $27.7 \%$ (10 ítems de 36) de los ítems son aceptables (ítems 1, 2, 3, 14, 15, 16, 17, 28, 29 y 30).

Por otra parte, el índice de dificultad indicó que el 36\% (13 ítems de 36) de los ítems tiene una dificultad baja (ítems 1, 2, 8, 9, 10, 13, 18, 24, 25, 28, 29, 30 y 34), el 47\% (17 ítems de 36) de los ítems tiene una dificultad media (ítems 3, 4, 5, 6, $7,14,15,16,17,19,20,21,22,23,27,33,35)$ у el 16\% (6 ítems de 36) dificultad alta $(11,12,26$, 31, 32, 36; véase la Tabla 2).

Validez de los percentiles propuestos. De manera general, la coincidencia al clasificar a los niños entre el punto de corte cubano (Ramírez-Benítez et al., 2015) y los percentiles propuestos es adecuada (69\%); no obstante, los valores de la sensibilidad son ligeramente bajos (véase la Tabla 3 ) debido a la cantidad de verdaderos positivos con respecto a la cantidad de falsos negativos (casos positivos según el punto de corte cubano y negativos por los percentiles propuestos).

A los 4 años la coincidencia resultó ligeramente baja (53\%), pues la sensibilidad resultó muy baja con varios falsos negativos $(46 \%$ de la 
Tabla 3

Sensibilidad, especificidad y coeficiente de probabilidad diagnóstica de los percentiles propuestos según el punto de corte cubano.

\begin{tabular}{lcccccccc}
\hline Edad & Sensibilidad & Especificidad & CPD + & CPD - & VN & VP & FN & FP \\
\hline 4 años & .23 & 1 & .23 & .77 & 79 & 30 & 96 & 0 \\
5 años & .48 & 1 & .48 & .52 & 66 & 67 & 72 & 0 \\
6 años & .50 & 1 & .50 & .50 & 110 & 144 & 139 & 0 \\
Total & .43 & 1 & .43 & .57 & 255 & 241 & 307 & 0 \\
\hline
\end{tabular}

Nota. CDP+ (coeficiente de probabilidad diagnóstica positivo), CPD- (coeficiente de probabilidad diagnóstica negativo), VN (verdaderos negativos), VP (verdaderos positivos), FN (falsos negativos) y FP (falsos positivos).

Tabla 4

Cantidad de niños clasificados según los percentiles propuestos en la submuestra con alteraciones en el neurodesarrollo (130 niños) de Ramírez-Benítez et al., 2015.

\begin{tabular}{llllll}
\hline \multicolumn{1}{c}{ Punto de corte cubano } & & \multicolumn{5}{c}{ Percentiles propuestos } \\
\hline $\mathbf{4}$ años & $\mathbf{P 1 0}(10)$ & $\mathbf{P 2 5}(11-13)$ & $\mathbf{P 5 0}(14)$ & $\mathbf{P 7 5}(15)$ & $\mathbf{P 9 0}(16)$ \\
Positivos $(\leq 14)$ & - & 3 & - & - & - \\
Negativos $(\geq 15)$ & & - & $12^{*}$ & 10 & 5 \\
$\mathbf{5}$ años & $\mathbf{P 1 0}(11)$ & $\mathbf{P 2 5}(12-14)$ & $\mathbf{P 5 0}(15-16)$ & $\mathbf{P 7 5}(17)$ & $\mathbf{P 9 0}(18)$ \\
Positivos $(\leq 16)$ & - & 35 & - & - & - \\
Negativos $(\geq 17)$ & - & - & $13^{*}$ & 2 & - \\
6 años & $\mathbf{P 1 0}(11-12)$ & $\mathbf{P 2 5}(13-15)$ & $\mathbf{P 5 0}(16-17)$ & $\mathbf{P 7 5}(18-19)$ & $\mathbf{P 9 0}(20)$ \\
Positivos $(\leq 17)$ & - & 23 & - & - & - \\
Negativos $(\geq 18)$ & - & - & $18^{*}$ & 8 & 1 \\
Total & $\mathbf{P 1 0}$ & $\mathbf{P 2 5}$ & $\mathbf{P 5 0}$ & $\mathbf{P 7 5}$ & $\mathbf{P 9 0}$ \\
Positivos & - & $61(46.92 \%)$ & - & - & - \\
Negativos & - & - & $43^{*}(33.07 \%)$ & $20(15.38 \%)$ & $6(4.61 \%)$ \\
\hline
\end{tabular}

Nota. Se han señalado con (*) los falsos negativos según el punto de corte de Ramírez-Benítez et al. (2015). Positivos: niños clasificados con alteraciones en el desarrollo. Negativos: niños clasificados sin alteraciones en el desarrollo según los percentiles propuestos.

muestra; véase la Tabla 3). A los 5 años la coincidencia mostró valores adecuados $(65 \%)$, aunque la sensibilidad se mantuvo baja debido a la cantidad de falsos negativos (34\% de la muestra). A los 6 años la coincidencia resultó adecuada (64\%) y en igual sentido la sensibilidad resultó apropiada tal y como se muestra en la Tabla 3, aunque con varios falsos negativos (35\% de la muestra).

En relación con el coeficiente de probabilidad diagnóstica, los resultados indicaron que los percentiles propuestos tienen un descenso pequeño de la probabilidad diagnóstica, ya sea en el $\mathrm{CPD}+$ como en el CPD-, tal y como se muestra en la Tabla 3. Este resultado ocurre cuando se realiza el análisis en la muestra total y por edades.

De manera general, el $46.92 \%$ de la submuestra con alteraciones en el neurodesarrollo fue clasificado por el percentil 25 , tal y como se muestra en la Tabla 4, el 33.07\% fue clasificado por el percentil 50, el $15.38 \%$ fue clasificado por 
el percentil 75 y el $4.61 \%$ por el percentil 90 . En la submuestra no hubo niños clasificados en el percentil 10. En cambio, el punto de corte cubano clasificó de la siguiente manera: $80 \%$ con bajo rendimiento intelectual y el $20 \%$ sin alteraciones.

\section{Discusión}

En la presente investigación, el análisis estadístico con una muestra mayor posibilitó apreciar un ajuste adecuado en las normativas cubanas del TMCR para niños en edad preescolar (de entre 4 y 6 años). En un estudio anterior, RamírezBenítez et al. (2015) ofrecieron un punto de corte para clasificar al niño en edad preescolar en dos grupos: niños con baja habilidad versus niños con habilidades típicas de la edad. Sin embargo, el resultado obtenido en este estudio ofrece una clasificación en cinco grupos: (1) niños con muy baja habilidad en el razonamiento analógico visual (percentil 10), (2) niños con baja habilidad (percentil 25), (3) niños con un desarrollo típico para la edad (percentil 50), (4) niños con alta habilidad (percentil 75) y (5) niños con habilidad superior (percentil 90).

La validez de los percentiles propuestos resultó adecuada cuando se la comparó con el punto de corte obtenido por Ramírez-Benítez et al. (2015). Además, el coeficiente de probabilidad diagnóstica indicó un descenso pequeño de la probabilidad diagnóstica de los percentiles obtenidos con respecto al punto de corte cubano.

En el primer resultado, la coincidencia diagnóstica entre los percentiles propuestos y el punto de corte cubano es del 69\%. El 31\% de los casos son considerados falsos negativos, pues los valores del percentil 50, obtenido por este estudio, son menores que los valores del punto de corte de Ramírez-Benítez et al. (2015). En consecuencia, un grupo de casos clasificados con bajo rendimiento intelectual por el punto de corte cubano son clasificados como negativos por los percentiles propuestos. La caída o la elevación de los valores de los puntajes es uno de los principales problemas que encuentran los investigadores cuando ofrecen percentiles y los comparan con otros percentiles. Por ende, cada investigador debe estimar los beneficios y dificultades que la propuesta ofrece. Tal es el caso de los estudios de Rossi-Casé et al. $(2015,2016)$ cuando comparan los puntajes de los percentiles 50 en diferentes años en una población de adolescentes argentinos (1964, 2000 y 2015). En especial, el percentil 50 en 2000 para adolescentes de entre 13 y 15 años fue de 48 puntos, mientras que el percentil 50 en el 2015 fue de 45 puntos. En este caso, la comparación entre las dos muestras puede generar varios falsos negativos, lo cual no fue informado en el estudio, pues la intención de los autores era demostrar el efecto Flynn. De esta forma, se recomienda realizar un análisis de beneficios y dificultades diagnósticas cuando se ofrece una nueva normativa. En este caso, el CPD (coeficiente de probabilidad diagnóstica) es una alternativa para estimar el efecto de los percentiles en una determinada muestra.

El presente estudio, basándose en los resultados del CPD, reveló que los percentiles propuestos tienen un pequeño descenso en la probabilidad diagnóstica con respecto al punto de corte cubano. Desde esta perspectiva, y considerando los resultados de la coincidencia al clasificar, se recomienda precaución al utilizar los percentiles en la práctica, pues se requieren otros estudios para verificar sus beneficios y dificultades. No obstante, cuando se realizó la clasificación de ambos puntos de corte en la muestra de niños con dificultades en el neurodesarrollo (submuestra de Ramírez-Benítez et al., 2015), se pudo observar el impacto práctico de los percentiles propuestos. En especial, el punto de corte cubano indicó muy pocos casos de niños con rendimiento inte- 
lectual típico para la edad (20\%), lo cual es destacable, pues los problemas del neurodesarrollo tienen una incidencia en el desarrollo de la inteligencia, pero no de manera tan significativa como para diagnosticar o suponer alteración intelectual en el $80 \%$ de la muestra, menos aún si se considera que los autores tomaron casos de niños con alteraciones del neurodesarrollo sin discapacidad intelectual severa.

En este caso, los percentiles propuestos son más flexibles al clasificar los casos en la submuestra de Ramírez-Benítez et al. (2015), pues si bien los niños con problemas del neurodesarrollo, y en dependencia de otros factores clínicos y educativos, presentan bajo rendimiento intelectual (tal y como se indica en la Tabla 4, percentil 25; 46.92\% de la submuestra), no aparece ningún niño clasificado por el percentil 10, lo cual indica que el bajo rendimiento intelectual en esta población no es tan significativo como se esperaba. Incluso se pudieron observar en los análisis algunos casos de niños clasificados en los percentiles 75 y 90 .

Desde esta perspectiva, los percentiles propuestos pueden diferenciar a los niños con bajo rendimiento intelectual; ya sea por inmadurez en su neurodesarrollo debida a diversos factores (percentil 25) o por factores más relacionados con lo neurogenético, con muy bajos valores en el rendimiento intelectual (percentil 10). En igual sentido, estos pueden determinar si los niños no presentan dificultades en su rendimiento intelectual o si presentan valores superiores (percentiles 75 y 90) no esperados, aún cuando tengan alteraciones en el neurodesarrollo.

En relación con las propiedades psicométricas de la prueba Raven, en la literatura se han informado varias investigaciones que aseveran la validez y confiabilidad de la prueba, ya sea a través de la Teoría Clásica del Test (TCT; FloresMendoza, Widaman, Bacelar, \& Lelé, 2014; Ibrahim \& Kazem, 2013; Mansilla et al., 2012;
Van der Elst et al., 2013) así como en la Teoría de la Respuesta al Ítem (TRI; Escurra-Mayaute \& Delgado-Vásquez, 2010; Sisto, Marín-Rueda, \& Bartholomeu, 2006).

En el caso del estudio de Escurra-Mayaute y Delgado-Vásquez (2010), los investigadores verificaron las propiedades psicométricas de la prueba Raven según las dos teorías (TCT y TRI) en una población de jóvenes universitarios. Con respecto a la TCT, la prueba mostró una consistencia interna adecuada (coeficiente Kuder-Richardson 20 con un valor de .79). En relación con la TRI, los primeros 20 ítems de la prueba fueron fáciles para los jóvenes, mientras que los demás indicaron niveles de dificultad entre adecuado y difícil. El índice de discriminación reveló que todos los ítems tienen un nivel de discriminación entre bueno y adecuado, aunque el ítem 13 no mostró buena discriminación. De esta forma, los autores aseveraron que el análisis de ítems es una forma apropiada de determinar las debilidades y fortalezas de la prueba con respecto a la dificultad del ítem y su capacidad para discriminar la habilidad a evaluar en una población dada.

En este sentido, en la presente investigación se realizó un análisis de ítems para evaluar las propiedades psicométricas de la prueba Raven, así como saber si la mayoría de los ítems son fáciles o difíciles para los niños en edad preescolar y si estos discriminan entre niños con alta y baja habilidad.

En este caso, el coeficiente alfa de Cronbach cuando el ítem es eliminado indicó valores adecuados, entre .84 y .89, en la muestra general. En igual sentido los valores de alfa por edades fueron significativos. No obstante, los ítems con posibilidad de aumentar alfa, ya sea de manera general o por edades, se destacan por ser más fáciles o más difíciles para el niño preescolar, tal es el caso de los ítems 1, 4 y 32, los dos primeros por ser más fáciles y el último por ser más complejo. A pe- 
sar del hecho de que haya varios ítems clasificados como difíciles o fáciles para los preescolares, la consistencia interna de la prueba es adecuada, tal y como se ha informado en varias investigaciones, ya sea a través de métodos test-retest (Mansilla et al., 2012; Rossi-Casé et al., 2015) o mediante el método de alfa de Cronbach (OssaCornejo, Jara-Quezada, \& Troncoso-San Martin, 2014; Ramírez-Benítez, Lorenzo-González, \& Díaz-Bringas, 2013).

De igual manera, este argumento puede ser confirmado por la correlación ítem-total, pues los ítems aportan valores a la varianza total de la prueba de manera significativa. Los valores de las correlaciones ítem-total son de entre .33 y .56 , es decir entre buenos y excelentes, según la clasificación de Ebel y Frisbie (1986).

De esta manera, se espera que la prueba identifique a niños con baja y alta habilidad en el razonamiento analógico. En efecto, el 100\% de los ítems mostraron un índice de discriminación entre bueno y aceptable. Por ende, la prueba es adecuada para discriminar entre niños con baja y alta habilidad. En este sentido, la prueba Raven puede ser aplicada en edad preescolar, en especial en niños de entre 4 años y 5 meses y 6 años y 10 meses, pues en esa edad el niño puede resolver los problemas de matrices y al mismo tiempo es posible clasificar el caso de cada niño en uno de cinco grupos diagnósticos según el resultado obtenido.

A manera de conclusión, en los niños en edad preescolar es necesario conocer las potencialidades y debilidades del desarrollo, pues esto posibilita trazar una estrategia de estimulación acorde con las necesidades de cada niño. El TM$\mathrm{CR}$ es una alternativa valiosa para el profesional de la educación y la salud, ya que es una prueba fácil de aplicar y sobre todo posibilita conocer el desarrollo del pensamiento del niño. De esta forma, los percentiles que ofrece este trabajo se establecen con la intención de identificar de ma- nera temprana las dificultades en el pensamiento para organizar intervenciones educativas y evitar un posible fracaso escolar relacionado con dificultades en la inteligencia fluida. No obstante, se recomienda realizar otras investigaciones para validar los percentiles con una muestra en la que se controlen las alteraciones del neurodesarrollo, ya sean síndromes genéticos o síndromes multifactoriales. En igual sentido, sería oportuno evaluar una muestra de niños en edad preescolar y valorar el impacto de la clasificación en la edad escolar, pues se espera que los niños clasificados dentro del percentil 25 no tengan el mismo desempeño escolar que aquellos clasificados en el percentil 10. Además, se podría valorar el rendimiento de los falsos negativos obtenidos por los percentiles propuestos con respecto al punto de corte de Ramírez-Benítez et al. (2015). Sería de importancia determinar hasta qué punto se trata en estos casos de falsos negativos.

\section{Conclusiones}

1. El análisis de ítems (alfa cuando el ítem es eliminado, correlación ítem-total e índices de discriminación y dificultad) indicó que el Test de Matrices Progresivas Coloreadas de Raven presenta propiedades psicométricas adecuadas para aplicarse en niños en edad preescolar, en especial en las edades comprendidas entre 4 y 6 años.

2. El análisis de coincidencia entre los percentiles propuestos y el punto de corte cubano y el análisis con el coeficiente de probabilidad diagnóstica indicaron que los percentiles propuestos deben utilizarse en la práctica con precaución. No obstante, la clasificación de los percentiles en una muestra de niños con alteraciones en el neurodesarrollo es más flexible y operativa que el punto de corte que ofrecen Ramírez-Benítez et al. (2015). 


\section{Referencias}

Anstey, E. (1963). Test de Dominós. Manual. Buenos Aires: Paidós.

Bandeira, D. R., Boccato-Alves, I. C., Giacomel, A. E., \& Lorenzatto, L. (2004). Matrizes Progressivas Coloridas de Raven - Escala Especial: Normas para Porto Alegre, RS. Psicologia em Estudo, 9(3), 479486. doi: 10.1590/s1413-73722004000300016

Bandeira, D. R., Costa, A., \& Arteche, A. (2012). The Flynn effect in Brazil: Examining generational changes in the Draw-a-Person and in the Raven's Coloured Progressive Matrices. Revista Latinoamericana de Psicología, 44(3), 9-18. Recuperado de http:// www.scielo.org.co/scielo.php?script=sci_arttex$\mathrm{t} \& \mathrm{pid}=\mathrm{S} 0120-05342012000300001 \& \operatorname{lng}=\mathrm{en} \& \mathrm{tl}-$ $\mathrm{ng}=\mathrm{en}$

Brouwers, S. A., Van de Vijver, F. J. R., \& Van Hemert, D. A. (2008). Variation in Raven's Progressive Matrices scores across time and place. Learning and Individual Differences, 19(3), 330-338. doi: 10.1016/j.lindif.2008.10.006

Cairo-Valcárcel, E., Cairo-Martínez, E., Bouza, C., \& Solozabal, T. P. (2000). Algunas características y posibilidades del Test de Matrices Progresivas de Raven. Revista Cubana de Psicología, 17(2), 95 -105 .

Cattell, R. B., \& Cattell, A. K. S. (1994). Tests de Factor «g», Escalas 2 y 3. Madrid: TEA Ediciones, S. A.

Crocker, L., \& Algina, J. (1986). An introduction to classical and modern test theory. New York: Holt, Rinehart \& Winston.

Crone, E., Wendelken, C., Van Leijenhorst, L., Honomichl, R., Christoff, K., \& Bunge, S. (2009). Neurocognitive development of relational reasoning. Developmental Science, 12(1), 55-66. doi: 10.1111/j.1467-7687.2008.00743.x

Ebel, R. L., \& Frisbie, D. A. (1986). Essentials of Education Measurement. Englewood Cliffs, NJ: Prentice Hall.

Escurra-Mayaute, L. M., \& Delgado-Vásquez, A. E. (2010). Análisis psicométrico del Test de Matrices
Progresivas Avanzadas de Raven mediante el modelo de tres parámetros de la teoría de la respuesta al ítem. Persona, 13, 71-97. Recuperado de https://revistas. ulima.edu.pe/index.php/Persona

Fernández-Liporace, M., Ongarato, P., Saavedra, E., \& Casullo, M. M. (2004). El Test de Matrices Progresivas, Escala General: Un análisis psicométrico. Evaluar, 4, 50-69. Recuperado de https://revistas. unc.edu.ar/index.php/revaluar

Fernández-Nistal, M. T., \& Mercado-Ibarra, S. M. (2014). Normative data of Raven's Coloured Progressive Matrices in Yaqui indigenous children. Anuario de Psicologia/The UB Journal of Psychology, 44(3), 373-385. Recuperado de http://revistes.ub.edu/index. php/Anuario-psicologia

Flores-Mendoza, C., Widaman, K. F., Bacelar, T. D., \& Lelé, Á. J. (2014). Propriedades psicométricas do Raven Geral no contexto de Minas Gerais. Arquivos Brasileiros de Psicologia, 66(2), 1-16. Recuperado de http://pepsic.bvsalud.org/scielo.php?script=sci_issuetoc\&pid=1809-526720140002\&lng=pt\&nr$\mathrm{m}=$ iso

Flynn, J. R. (2013). The "Flynn Effect" and Flynn's paradox. Intelligence, 41(6), 851-857. doi: 10.1016/j. intell.2013.06.014

Flynn, J. R., \& Rossi-Casé, L. (2012). IQ gains in Argentina between 1964 and 1998. Intelligence, 40(2), 145150. doi: 10.1016/j.intell.2012.01.006

IBM (2012). SPSS Statistics 21.0.0. [software de cómputo]. Disponible en https://www-01.ibm.com/software/ $\mathrm{mx} /$ analytics/spss/products/statistics

Ibrahim, A. M., \& Kazem, A. M. (2013). Psychometric properties of scores from an embedded and independently-administered short form of the Raven's Advanced Progressive Matrices. International Journal of Learning Management Systems, 1(2), 25-35. doi: 10.12785/ijlms/010203

Ivanovich, R., Forno, H., Durán, M. C., Hazbún-Game, J., Castro, J., \& Ivanovich, C. (2000). Estudio de la capacidad intelectual (Test de Matrices Progresivas de Raven) en escolares chilenos de 5 a 18 años. 
Antecedentes generales, normas y recomendaciones. Revista de Psicología General y Aplicada, 53(1), 5-30. Recuperado de https://dialnet.unirioja.es/ servlet/articulo? codigo $=2356766$

Lázaro, J., Ostrosky, F., \& Lozano, A. (2012). Batería Neuropsicológica de Funciones Ejecutivas y Lóbulos Frontales-2. Manual Moderno: México.

Mansilla, C., Vásquez, D., \& Estrada, C. (2012). Pertinencia normativa del Raven para la evaluación de población infantojuvenil socialmente vulnerable. Terapia Psicológica, 30(1), 73-80. doi: 10.4067/ s0718-48082012000100007

Ministerio de Salud Pública, Cuba. (2013). Dirección nacional de registros médicos y estadísticas de salud. Anuario estadístico de salud 2013. Recuperado de files.sld.cu/dne/files/2014/05/anuario-2013-esp-e.pdf Moratalla-Rodríguez, G. (2015). Lectura crítica de artículos de pruebas diagnósticas II: Análisis de resultados. Radiología, 57, 22-28. doi: 10.1016/j.rx.2014.11.004

Neisser, U., Boodoo, G., Bouchard, T. J. Jr., Boykin, A. W., Brody, N., Ceci, S. J. ... Urbina, S. (1996). Intelligence: Knowns and unknowns. American Psychologist, 51(2), 77-101. doi: 10.1037/0003-066x.51.2.77

Ossa-Cornejo, C. J., Jara-Quezada, J., \& Troncoso-San Martin, T. (2014). Validación Test de Matrices Progresivas de Raven Escala Coloreada, en escolares de la ciudad de Chillán. Memorias de Investigación. Recuperado de http://repobib.ubiobio.cl/jspui/ handle/123456789/204

Ramírez-Benítez, Y., Jiménez-Morales, R. M., \& DíazBringas, M. (2015). Matrices progresivas de Raven: Punto de corte para preescolares 4 - 6 años. Evaluar, 15, 123-133. Recuperado de https://revistas.unc.edu. ar/index.php/revaluar

Ramírez-Benítez, Y., Lorenzo-González, G., \& DíazBringas, M. (2013). Matrices progresivas de Raven y su versión abreviada en la población escolar. Revista Mexicana de Neurociencias, 14(2), 63-67. Recuperado de http://revmexneuroci.com/

Ramírez-Benítez, Y., Torres-Diaz, R., \& Amor-Diaz, V.
(2016). Contribución única de la inteligencia fluida y cristalizada en el rendimiento académico. Revista Chilena de Neuropsicología, 11(2), 1-5. Recuperado de http://www.neurociencia.cl

Raven, J. C. (2004). Test de Matrices Progresivas. Escala Coloreada. Cuaderno de Matrices / Series A, AB y B. México: Editorial Paidós.

Rossi-Casé, L., Neer, R., Lopetegui, S., Doná, S. M., Biganzoli, B., \& Garzaniti, R. (2014). Matrices Progresivas de Raven: Efecto Flynn y actualización de baremos. Revista de Psicología, 23(2), 3-13. doi: 10.5354/0719-0581.2014.36144

Rossi-Casé, L., Neer, R., Lopetegui, S., Doná, S. M., Biganzoli, B., \& Garzaniti, R. (2015). Test de Raven, baremos argentinos para el rango 13-18 años y efecto Flynn. Orientación y Sociedad, 15, 1-24. Recuperado de http://www.scielo.org.ar/scielo.php?script=sci_arttext\&pid=S1851-88932015000100005\&lng=es\&n$\mathrm{rm}=$ iso

Rossi-Casé, L., Neer, R., Lopetegui, S., Doná, S. M., Biganzoli, B., \& Garzaniti, R. (2016). Test de Raven: Actualización de baremos en adolescentes argentinos y análisis del efecto Flynn. Revista Iberoamericana de Diagnóstico y Evaluación, 42(2), 3-13. doi: 10.21865/RIDEP42_3

Sisto, F. F., Marín-Rueda, F. J., \& Bartholomeu, D. (2006). Estudo sobre a unidimensionalidade do Teste Matrizes Progressivas Coloridas de Raven. Psicologia: Reflexão e Critica, 19(1), 66-73. doi: 10.1590/S0102-79722006000100010

Solovieva, Y., Loredo, D., Quintanar, L., \& Lázaro, E. (2013). Caracterización neuropsicológica de una población infantil urbana a través de la Evaluación Neuropsicológica Infantil Puebla-Sevilla. Pensamiento Psicológico, 11(1), 83-98. Recuperado de http://revistas.javerianacali.edu.co/index.php/ pensamientopsicologico

Spearman, C. (1904). "General intelligence" objectively determined and measured. The American Journal of Psychology, 15(2), 201. doi: 10.2307/1412107

Sundet, J. M., Barlaug, D. G., \& Torjussen, T. M. (2004). 
The end of the Flynn effect? A study of secular trends in mean intelligence test scores of Norwegian conscripts during half a century. Intelligence, 32, 349362. doi: 10.1016/j.intell.2004.06.004

Teasdale, T. W., \& Owen, D. R. (2007). Secular declines in cognitive test scores: A reversal of the Flynn effect. Intelligence, 36, 121-126. doi: 10.1016/j. intell.2007.01.007

Tenorio, M., Arango, P., Aparicio, A., Benavente, C., Thibaut, C., \& Rosas, R. (2012). Test de Evaluación Neuropsicológica Infantil. Pontificia Universidad Católica de Chile.

Van der Elst, W., Ouwehand, C., Van Rijn, P., Lee, N., Van Boxtel, M., \& Jolles, J. (2013). The shortened Raven Standard Progressive Matrices: Item response theory-based psychometric analyses and normative data. Assessment, 20(1), 48-59. doi: 10.1177/1073191111415999

Wechsler, D. (2011). Manual de Administración y Puntuación (WISC-IV). Buenos Aires: Paidós.

Wechsler, D. (2012). Wechsler Preschool and Primary Scale of Intelligence (WPPSI-IV) ( $4^{\mathrm{a}}$ ed.). San Antonio, TX: Pearson. 